\title{
Implementation of a fiber Bragg grating sensor network for structural monitoring of a new stone bridge
}

\author{
L. A. Ferreira, F. M. Araújo \\ INESC Porto, Campus da FEUP, R. Dr. Roberto Frias, 378, 4200-465 Porto, Portugal \\ FiberSensing, R. Vasconcelos Costa, 277, 4470-640, Portugal \\ C. Barbosa, N. Costa \\ FiberSensing, R. Vasconcelos Costa, 277, 4470-640, Portugal
}

A. Arêde, A. Costa, P. Costa

Fac. Eng. Universidade do Porto, R. Dr. Roberto Frias, s/n, 4200-465 Porto, Portugal

\begin{abstract}
The implementation of a large sensing network based on fiber optic sensors for the structural health monitoring of a new five arch stone bridge with a total span of $60 \mathrm{~m}$ over Vizela River (Felgueiras, Portugal) is presented. In addition to the implemented instrumentation with conventional sensors, four different types of fiber Bragg grating based sensors were specially developed for measuring temperature, strain and displacement in several critical points of the structure. In total, 85 Bragg sensors were installed and more than $800 \mathrm{~m}$ of fiber cables were deployed along the bridge. The fiber optic sensing network installed on the bridge was entirely monitored by using a single measurement unit designed for collecting and archiving the signals from all the fiber Bragg grating sensors, and also to allow remote access to the important data through a standard GPRS connection.
\end{abstract}

\section{INTRODUCTION}

\subsection{Context}

As it is the case in many European countries, there is in Portugal a large number of bridges with stone arches. Such bridges are traditionally old and were not designed for the loads and traffic that are presently crossing them. Moreover, several bridges of this type show clear signs of structural degradation and lack of maintenance that may lead into situations where safety is not assured. Therefore, the possibility of monitoring and analyzing the structural behavior of a real bridge with stone arches represents an important contribution for the knowledge of this type of structures. The work presented here refers to the development and implementation of a fiber Bragg grating based sensing network for structural monitoring of a new stone bridge over Vizela River in Vila Fria, Felgueiras, Portugal, which was build to replace an old bridge with serious safety fragilities (Figure 1).
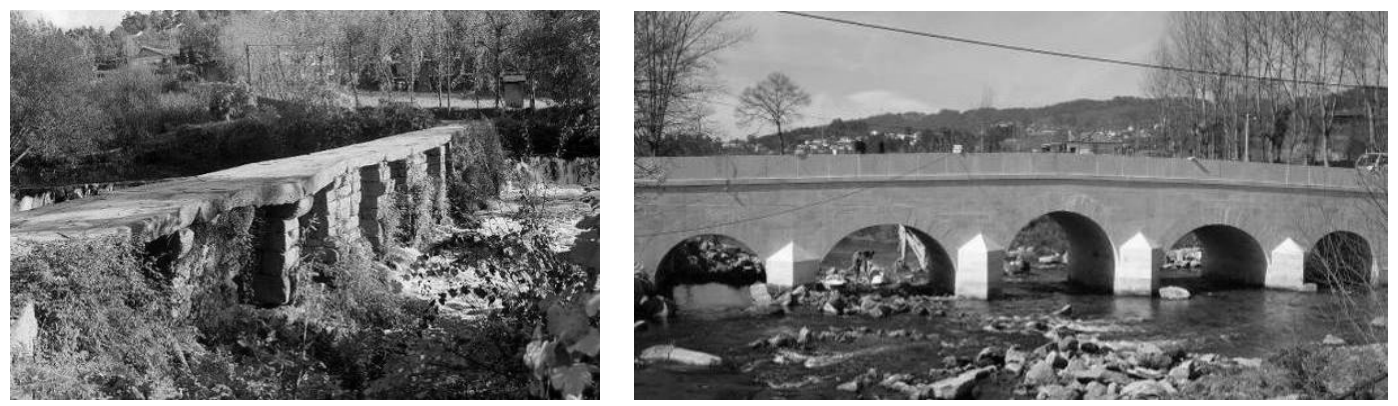

Figure 1. The Vila Fria (a) old and (b) new stone masonry bridge. 


\subsection{Why fiber optics?}

In general, modeling structures like the Vila Fria bridge brings several issues on how to define parameters to calibrate the mathematical models of the structure and on how to be certain about the results predicted by those models. Therefore, the possibility of monitoring an arch stone bridge from the beginning of its construction is of particular interest because it allows the installation of a large number of sensors according to a pre-established plan and accessing points impossible to achieve in structures that are already built. With the large amount of information that can be generated, improved calibration and models validation can be obtained. The fact that the bridge can then be monitored for a long period also allows the evaluation of its structural behavior and its relation to the usually observed pathologies in this kind of structures.

Nevertheless, the establishment of a large sensing network for monitoring multiple parameters that are structurally relevant is not always easy when using conventional electric technology. In fact, conventional sensors suffer from high EMI/RFI sensitivity, environment induced drift and require individual electrical stimulus that makes large scale structural health monitoring highly complex, particularly in hazard-environments common in Civil Engineering monitoring applications. Fiber optic sensors, and in particular, fiber Bragg gratings ${ }^{1}$, constitute a particularly appropriate and competitive alternative for structural health monitoring applications ${ }^{2,3}$. In fact, fiber Bragg grating sensors add to the long recognized advantages of fiber optic sensors (e.g., immunity to EMI/RFI, remote monitoring, small size and weight, electrical isolation, intrinsically safe operation, high sensitivity, long-term reliability) the inherent multiplexing capability and the ability to provide absolute measurements without the need for referencing ${ }^{4}$. Fiber Bragg grating technology is therefore becoming the natural substitute for the conventional sensing technologies by easing health monitoring of large structures during construction, load tests and long-term service ${ }^{5}$.

\subsection{Fiber optic monitoring project}

The monitoring of the Vila Fria stone masonry bridge pursued two main objectives: the establishment and calibration of analytical modeling techniques suitable for the numerical simulation of similar constructions; the evaluation and systematization of the main features of this type of structures in terms of their structural behavior and their relation with the most frequent structural pathologies. To achieve these goals, a vast instrumentation project was put forward to transform the bridge into a "live laboratory", which included, in addition to the installation of several conventional sensors described in reference 6, the deployment of a large fiber sensing network for the measurement of temperature (28 sensors), displacement (48 sensors) and strain (9 sensors). Some of the sensors used in the bridge were specifically developed by FiberSensing for this project, as it is the case of the LPDS - Linear Position and Displacement Sensor, and the Long Gauge. Most temperature sensors were used for referentiation of both fiber optic and conventional sensors.

The sensors were deployed in a tree network configuration with 15 branches. To interrogate this sensing network, a BraggMeter measurement unit containing an optical switch with 16 channels was adopted. In total, more than $800 \mathrm{~m}$ of fiber optic cables were installed in the bridge. Figure 2 shows a simplified scheme of the fiber optic sensing network architecture.

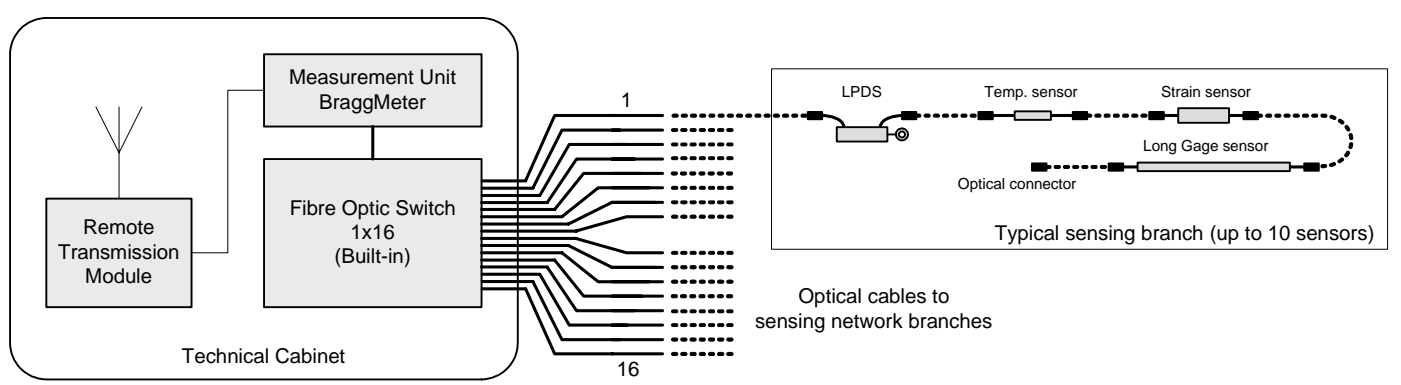

Figure 2. Implemented fiber optic sensing network. 


\section{THE FIBER OPTIC SENSING NETWORK COMPONENTS}

\subsection{LPDSs - Linear Position and Displacement Sensors}

To measure relative displacements between some stone blocks of the bridge, a Linear Position and Displacement Sensor based in a fiber Bragg grating was developed. The working principle of the sensor is quite common; it is based on the transduction of the displacement in force applied to the Bragg grating through the use of a division spring ${ }^{7,8}$. The fiber Bragg grating works therefore as a dynamometer. Nevertheless, the mechanical construction of this sensor is highly demanding, as can be seen from the CAD drawing in Figure 3. In fact, in order to obtain linear behavior over the desired measurement range and avoid hysteretic behavior, it is extremely important to eliminate any internal sources of friction. Also, to allow the sensor to be used in series and fixed to the structure as a conventional LVDT, it is necessary to design it in such a way that the input and output fibers enter and exit the device outside the longitudinal axis, which implies bending the fiber near the dynamometer moving parts. Adding to this, the need to make the sensor robust and hermetic places many constrains in the selection of materials as well as in their processing.

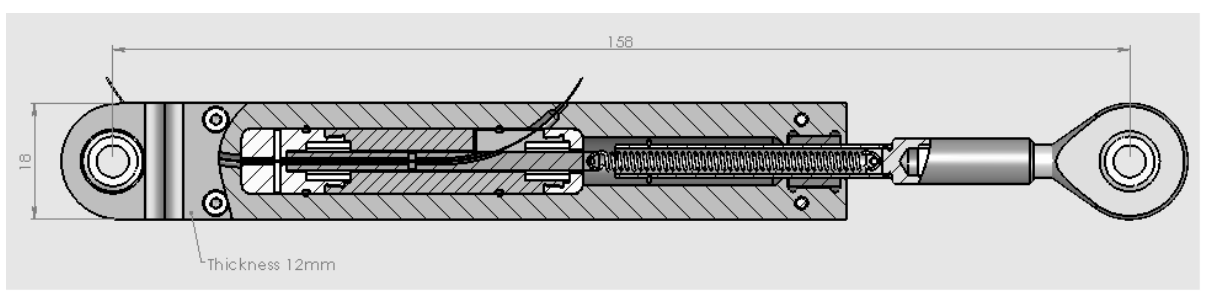

Figure 3. CAD drawing of the LPDS.

Figure 4 shows the final aspect of the developed sensor and Table 1 summarizes its characteristics. In Figure 5 it is shown a typical calibration cycle. It can be seen that the response of the sensor is linear and no hysteretic behavior is observable.

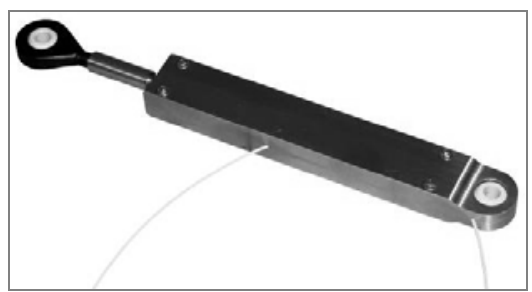

Figure 4. Final aspect of the LPDS.

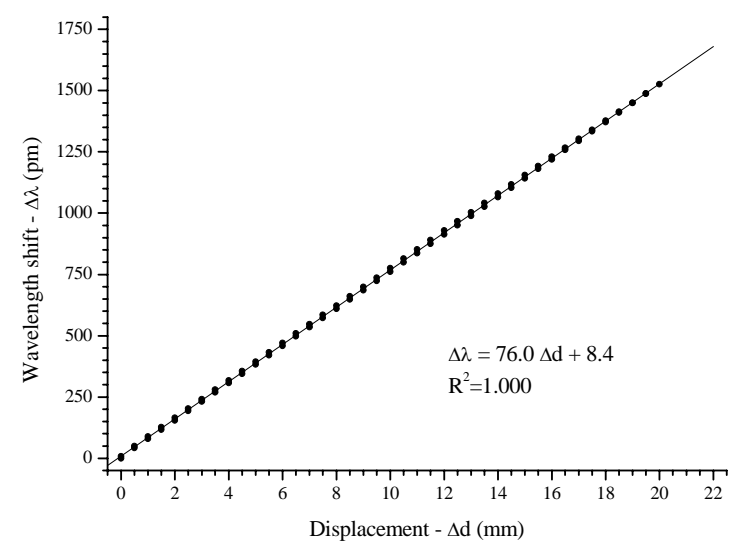

Figure 5. Typical calibration cycle for the LPDS. 
Table 1. Main specifications of the LPDS.

\begin{tabular}{ll}
\hline Optical & \\
\hline central wavelength & $\mathrm{C}$ band $(1530 \mathrm{~nm}$ to $1570 \mathrm{~nm})$ \\
spectral width (FWHM) & $<0.2 \mathrm{~nm}$ \\
reflectivity & $>75 \%$ \\
insertion loss & $<0.1 \mathrm{~dB}$ \\
side lobe suppression & $>10 \mathrm{~dB}$ \\
sensitivity & $76 \mathrm{pm} / \mathrm{mm}$ \\
maximum measurement range & $\pm 12.5 \mathrm{~mm}$ \\
\hline Mechanical & \\
\hline packaging & stainless steel \\
dimensions & $12 \mathrm{~mm} \times 18 \mathrm{~mm}$ x $205 \mathrm{~mm}$ (max.) \\
distance between anchoring points & $170 \pm 5 \mathrm{~mm}$ (adjustable) \\
weight & $200 \mathrm{~g}$ \\
\hline Environmental & \\
\hline operation temperature & $-20{ }^{\circ} \mathrm{C}$ to $80^{\circ} \mathrm{C}$ \\
relative humidity & $<90 \%$ at $40^{\circ} \mathrm{C}$ (no condensation) \\
\hline Inputs / Outputs & \\
\hline buffer & $\varnothing 900 \mu \mathrm{m}$ \\
length & $1 \mathrm{~m}$ \\
connectors & FC/PC
\end{tabular}

On the bridge, 44 LPDSs were used to measure the opening and closing of the joints between selected stone blocks of the face of the arches - in a standard Z configuration, Figure 6 - and also in the intrados of the arches - in the longitudinal and transverse directions. In each $\mathrm{Z}$ set, and also in each intrados set, a temperature sensor (described below) was used to compensate for temperature cross-effects, accordingly to Table 2. For aesthetic reasons, special cavities were formed in the stone blocks to hide the sensors, as can be seen in Figure 6. All the fiber optic connections were made from inside during the bridge construction or immersed in the mortar used to fill the joints.
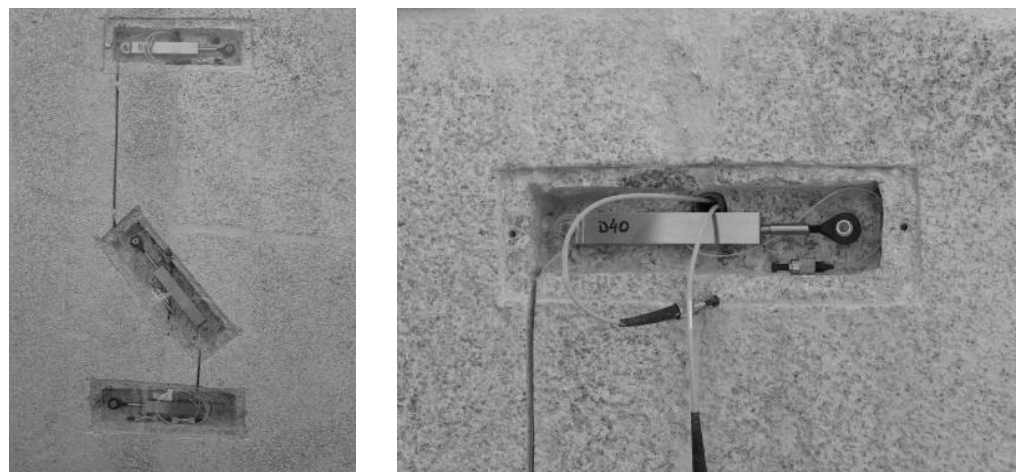

Figure 6. Installation of the LPDSs: (a) Z configuration; (b) detail of the stone housing.

Table 2. Association between sets of LPDSs and temperature sensors.

\begin{tabular}{cc}
\hline Arches face $(\mathrm{Z})$ & \\
\hline D01, D02, D03 & T1 \\
D04, D05, D06 & T2 \\
D07, D08, D09 & T3 \\
D10, D11, D12 & T4 \\
D13, D14, D15 & T5 \\
D16, D17, D18 & T6 \\
D19, D20, D21 & T7 \\
D22, D23, D24 & T8 \\
D25, D26, D27 & T9 \\
D31, D32, D33 & T10 \\
D40, D41, D42 & T11 \\
\hline Intrados & \\
\hline D46, D47, D48 & T24 \\
D49, D50, D51, D52 & T26 \\
D53, D54, D55, D56 & T28 \\
\hline
\end{tabular}




\subsection{Temperature sensors}

Temperature sensors used in the bridge are shown in Figure 7. Table 3 summarizes their characteristics and in Figure 8, a calibration line is presented. As mentioned previously, they were mainly used to compensate for temperature cross effects, not only for the fiber optic sensors but also for other type of sensors used in the bridge. Table 4 lists the temperature sensors that were used in combination with conventional differential fluid pressure sensors for level measurements (level sensors).

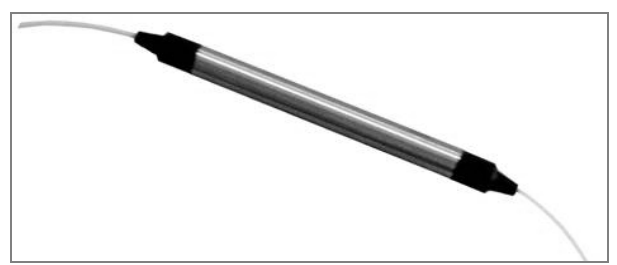

Figure 7. Temperature sensor.

Table 3. Main specifications of the temperature sensor.

\begin{tabular}{ll}
\hline Optical & C band $(1530 \mathrm{~nm}$ to $1570 \mathrm{~nm})$ \\
\hline central wavelength & $<0.2 \mathrm{~nm}$ \\
spectral width (FWHM) & $>75 \%$ \\
reflectivity & $<0.1 \mathrm{~dB}$ \\
insertion loss & $>10 \mathrm{~dB}$ \\
side lobe suppression & $29.2 \mathrm{pm} /{ }^{\circ} \mathrm{C}$ \\
sensitivity & $-10^{\circ} \mathrm{C}$ to $80^{\circ} \mathrm{C}$ \\
maximum measurement range & \\
\hline Mechanical & stainless steel \\
\hline packaging & $\varnothing 6.3 \mathrm{~mm} \times 56.5 \mathrm{~mm}$ \\
dimensions & $100 \mathrm{~g}$ \\
weight & \\
\hline Environmental & $-20{ }^{\circ} \mathrm{C}$ to $80^{\circ} \mathrm{C}$ \\
\hline operation temperature & $<90 \%$ at $40{ }^{\circ} \mathrm{C}$ (no condensation) \\
relative humidity & \\
\hline Inputs / Outputs & $\varnothing 900 \mu \mathrm{m}$ \\
\hline buffer & $1 \mathrm{~m}$ \\
length & FC/PC \\
connectors &
\end{tabular}

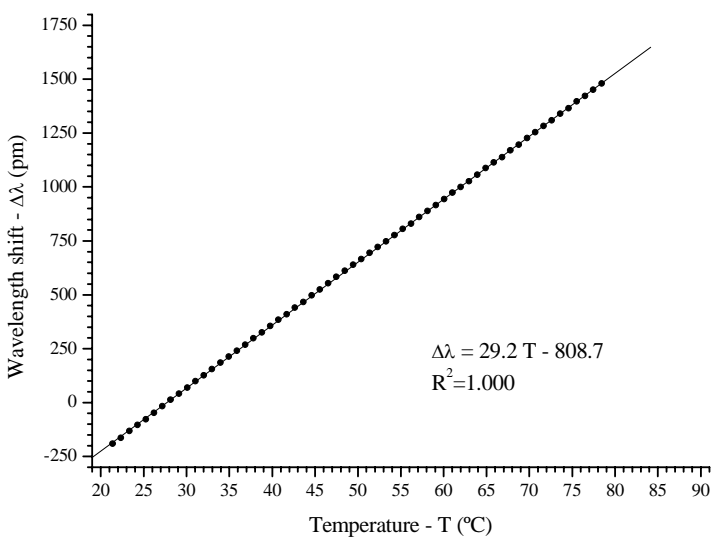

Figure 8. Calibration curve for the temperature sensor.

Table 4. Temperature sensors associated with non-fiber optic pressure sensors.

\begin{tabular}{cc}
\hline Level sensors & \\
\hline T12 & T13 \\
T14 & T15 \\
T16 & T17 \\
T18 & T19 \\
T20 & T21 \\
\hline
\end{tabular}




\subsection{Strain sensors}

Figure 9 shows an example of the sensors installed in the bridge for strain measurement. These sensors were produced by embedding pre-tensioned fiber Bragg gratings in composite materials, which provides a device that is very robust and easy to apply in field. Table 5 summarizes the main characteristics of this sensor. Nine sensors were installed in the arches of the bridge, mainly for the control of tension in the extrados and intrados, as indicated in Table 6. Figure 10 shows sets of two sensors being installed along two orthogonal directions.

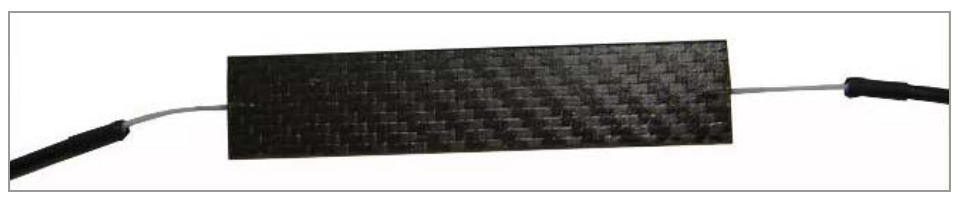

Figure 9. Composite strain sensor.

Table 5. Main specifications of the composite strain sensor.

\begin{tabular}{ll}
\hline Optical & \\
\hline central wavelength & $\mathrm{C}$ band $(1530 \mathrm{~nm}$ to $1570 \mathrm{~nm})$ \\
spectral width (FWHM) & $<0.2 \mathrm{~nm}$ \\
Reflectivity & $>75 \%$ \\
insertion loss & $<1 \mathrm{~dB}$ \\
side lobe suppression & $>10 \mathrm{~dB}$ \\
Sensitivity & $1.2 \mathrm{pm} / \mu \varepsilon$ \\
maximum measurement range & $\pm 2 \mathrm{~m} \varepsilon$ \\
\hline Mechanical & \\
\hline Packaging & stainless steel \\
Dimensions & $20 \mathrm{~mm} \times 100 \mathrm{~mm} \times 0.9 \mathrm{~mm}$ \\
Weight & $25 \mathrm{~g}$ \\
\hline Environmental & \\
\hline operation temperature & $-20^{\circ} \mathrm{C}$ to $80^{\circ} \mathrm{C}$ \\
relative humidity & $<90 \%$ at $40^{\circ} \mathrm{C}$ (no condensation) \\
\hline Inputs / Outputs & \\
\hline Buffer & $\varnothing 3 \mathrm{~mm} ; \varnothing 900 \mu \mathrm{m}$ \\
Length & $1 \mathrm{~m}$ \\
Connectors & FC/PC \\
\hline
\end{tabular}

Table 6. Composite strain sensors.

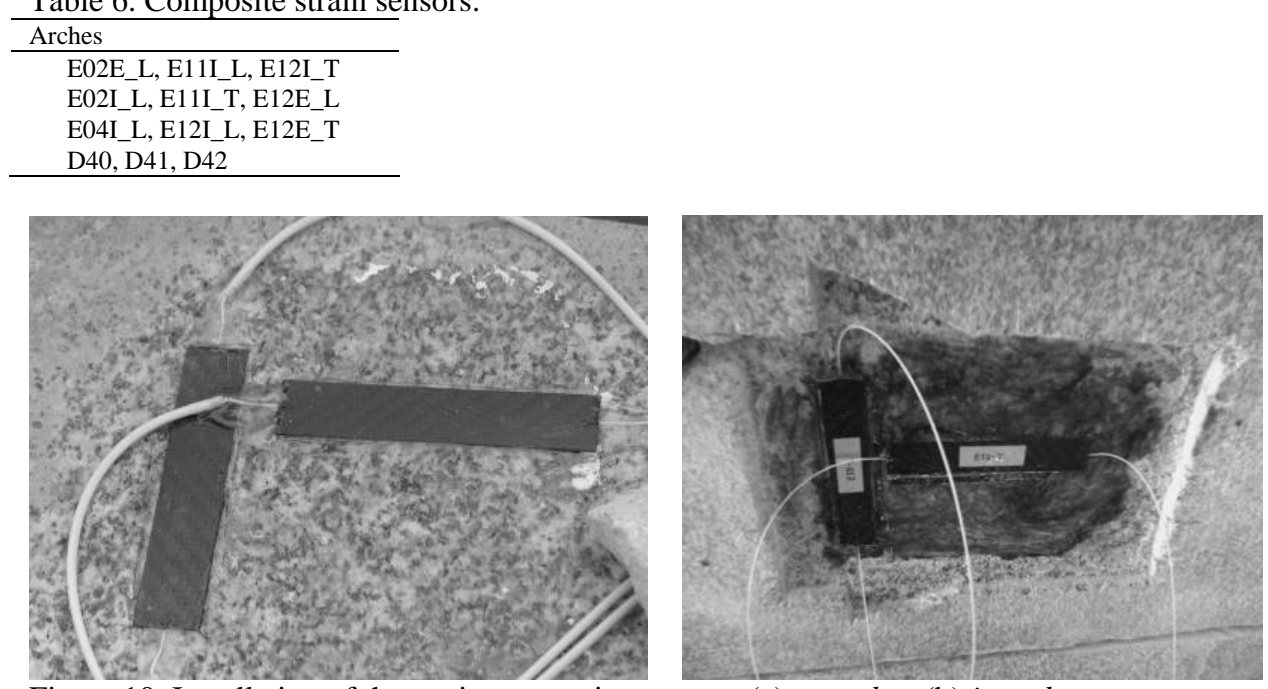

Figure 10. Installation of the strain composite sensors: (a) extrados; (b) intrados.

\subsection{Long Gauge sensors}

The long gauge sensor was also specially developed for this project. The objective was to measure small relative displacements between the bridge timpani walls that are separated by $\sim 4.7 \mathrm{~m}$. Basically, the displacement is measured through the measurement of deformation in an Invar 
wire with uniform cross-section $(\varnothing 1.27 \mathrm{~mm})$ that is fixed to each one of the timpani walls under an initial tension. To allow the installation of this sensor inside the soil, it was necessary to develop a suitable protection for the wire, as well as a mechanism for the application of tension in situ. The CAD drawing of the complete system shown in Figure 11 illustrates how those elements were build. The wire is protected by a set of three tubes; two of them, with $1 \mathrm{~m}$ length each, are threaded to the parts to be fixed to the stone blocks; a longer tube, $4 \mathrm{~m}$ long, is used outside these ones, and can be moved to allow later access to the wire fixing points and the tensioning mechanism, which is based on a secondary thread. Before the installation, a few tests were implemented in order to evaluate not only the sensor performance, but also the procedure of its installation. The results of one of those tests is shown in Figure 12, where it can be seen the agreement between values measured with the fiber Bragg grating based Long Gauge and those measured with a conventional LVDT.

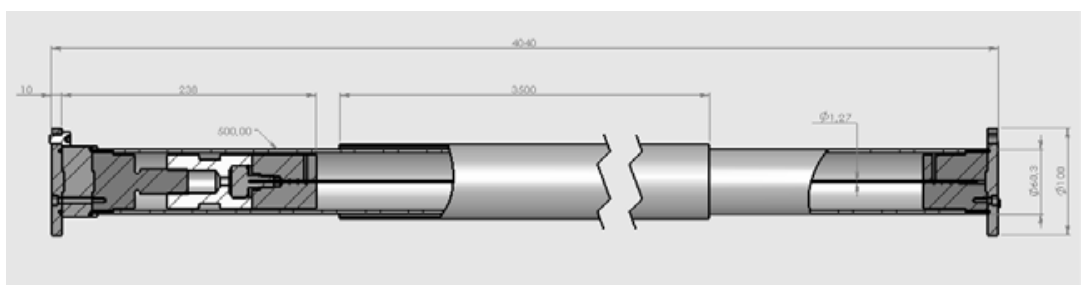

Figure 11. CAD drawing of the Long Gauge sensor.

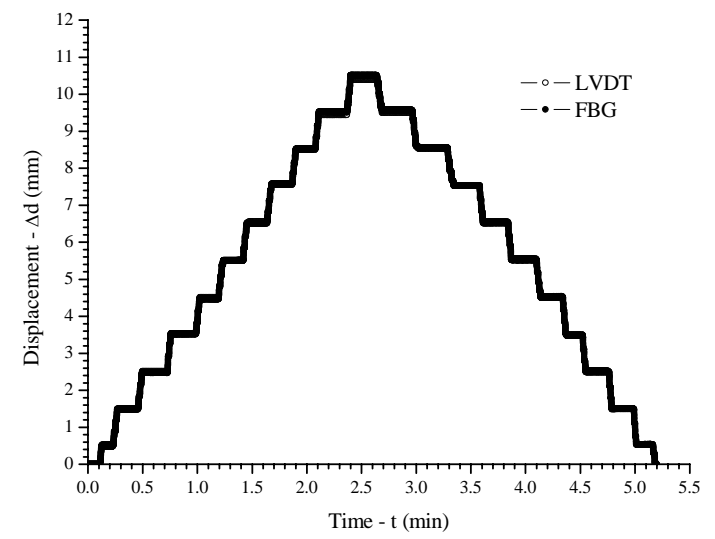

Figure 12. Laboratory testing of the Long Gauge.

Figure 13 shows the in situ installation of the Long Gauge sensors, where it can be seen the metallic parts used for fixing the wire to the stone blocks, the tensioning mechanism, the Invar wire, and the hermetic stainless steel tube that protects all the elements (Figure 13 (a)).
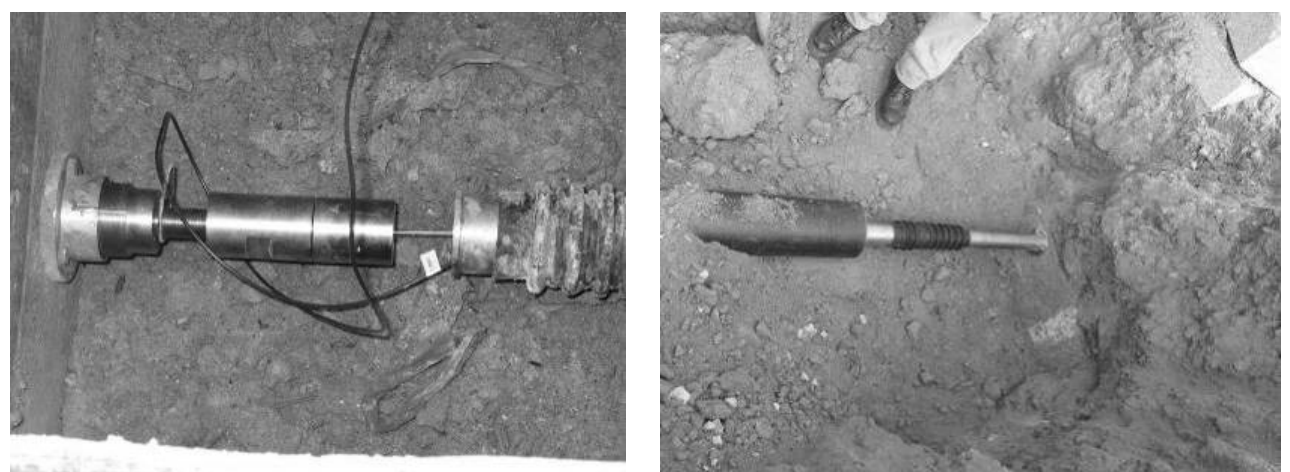

Figure 13. Installation of the Long Gauge sensors: (a) detail of the tension mechanism; (b) anchoring and protection. 
As an additional mean of protection, a secondary larger tube was used, thus ensuring that the entire system is immune to bending and vertical movements due to self weight and traffic loads (Figure 13 (b)). Table 7 lists the four Long Gauges installed in the bridge together with the associated compensation temperatures sensors.

Table 7. Association between sets of Long Gauge sensors and temperature sensors.

\begin{tabular}{cc}
\multicolumn{2}{c}{ Timpani walls } \\
\hline D61 & T22 \\
D62 & T23 \\
D63 & T25 \\
D64 & T27 \\
\hline
\end{tabular}

\subsection{Measurement unit}

The sensing network installed in the Vila Fria bridge was interrogated by using the BraggMeter measurement unit shown in Figure 14, which has an internal built-in optical switch with 16 channels. The unit has a sampling rate of $1 \mathrm{~S} / \mathrm{s}$ (for all the sensors in one channel) and the switching time is less than one second, which means that measurement data relative to all the sensors in the network can be collected every minute or less. Table 8 lists the most relevant characteristics of this measurement unit. A GPRS transmission module was connected to the unit in order to provide remote access to gathered data.

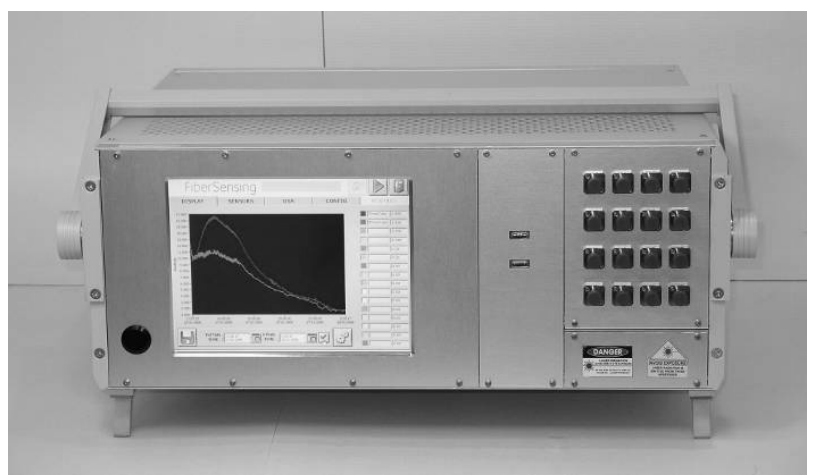

Figure 14. BraggMeter measurement unit.

Table 8. Main specifications of the BraggMeter used in the Vila Fria bridge.

Wavelength Measurement

\begin{tabular}{|c|c|}
\hline operating range & $\mathrm{C}$ band $(1530 \mathrm{~nm}$ to $1570 \mathrm{~nm})$ \\
\hline resolution & $0.5 \mathrm{pm}$ \\
\hline absolute accuracy & $\pm 2.0 \mathrm{pm}$ \\
\hline repeatability & $\pm 1.0 \mathrm{pm}$ \\
\hline sensors per fiber & 12 (for meas. ranges of $\sim 3 \mathrm{~nm}$ per sensor) \\
\hline acquisition rate & $1 \mathrm{~S} / \mathrm{s}$ \\
\hline \multicolumn{2}{|l|}{ aser Source } \\
\hline optical output power & $10 \mathrm{dBm}(\max )$ \\
\hline linewidth & $150 \mathrm{kHz}$ \\
\hline optical isolation & $60 \mathrm{~dB}$ \\
\hline \multicolumn{2}{|l|}{ nvironmental } \\
\hline operation temperature & $10^{\circ} \mathrm{C}$ to $40^{\circ} \mathrm{C}$ \\
\hline relative humidity & $<90 \%$ at $40{ }^{\circ} \mathrm{C}$ (no condensation) \\
\hline \multicolumn{2}{|l|}{ puts / Outputs } \\
\hline optical connectors & FC/PC \\
\hline instrument interface & touch screen, Ethernet, USB \\
\hline \multicolumn{2}{|l|}{ atalogger Functions } \\
\hline \multicolumn{2}{|c|}{ sampling, archiving, transmission, Excel ${ }^{\mathrm{TM}}$ compatible files } \\
\hline \multicolumn{2}{|l|}{ Iechanical } \\
\hline dimensions & $450 \mathrm{~mm} \times 180 \mathrm{~mm} \times 370 \mathrm{~mm}$ \\
\hline weight & $15 \mathrm{~kg}$ \\
\hline \multicolumn{2}{|l|}{ ower Requirements } \\
\hline voltage & $100-240 \mathrm{~V}$ \\
\hline power & $300 \mathrm{~W}$ \\
\hline frequency & $50-60 \mathrm{~Hz}$ \\
\hline
\end{tabular}




\begin{tabular}{ll}
\hline Optical Switch & \\
\hline channels & 16 \\
insertion loss & $0.5 \mathrm{~dB}$ \\
crosstalk & $-80 \mathrm{~dB}$ \\
back reflection & $-62 \mathrm{~dB}$ \\
polarization dependent loss & $0.02 \mathrm{~dB}$ \\
repeatability & $0.02 \mathrm{~dB}$ \\
switching time & $25 \mathrm{~ms}$ \\
internal optical fiber & singlemode, $8 / 125 \mu \mathrm{m}$ \\
\hline
\end{tabular}

\section{DISTRIBUTION OF THE SENSING NETWORK ALONG THE BRIDGE}

\subsection{Architecture of the network}

The instrumentation project is of great importance for maximizing the monitoring efficiency. The location of all the equipments and sensors must be carefully studied in order to obtain the maximum information with optimized resources. Thus the architecture must be defined taking into account singular spots that are particularly important in terms of structural behavior. Figure 15 shows a plan of the fiber optical monitoring project of the Vila Fria bridge.

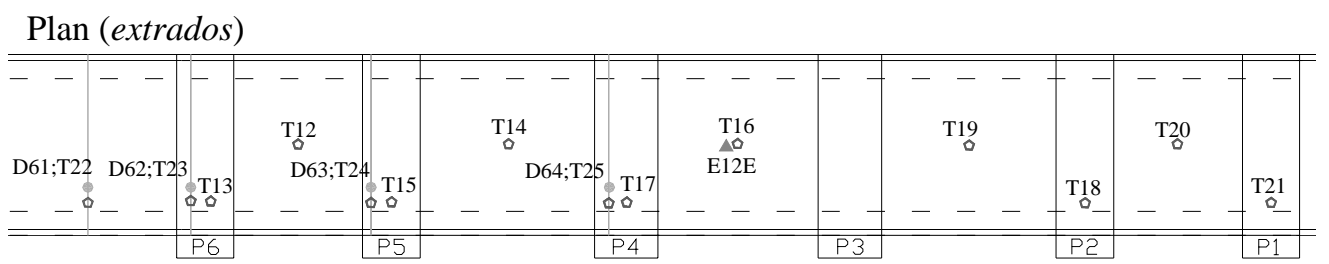

Downstream View

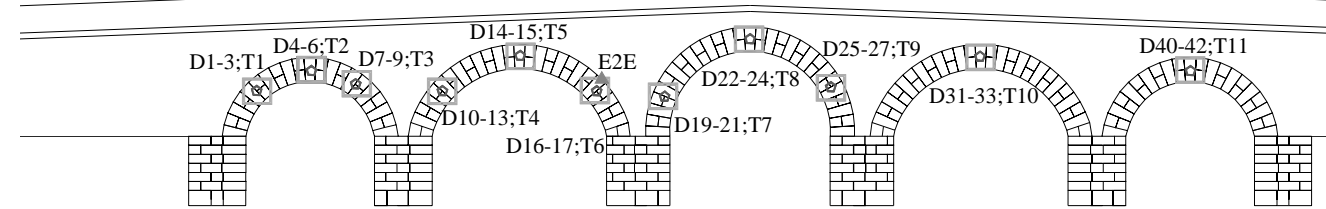

Plan (intrados)

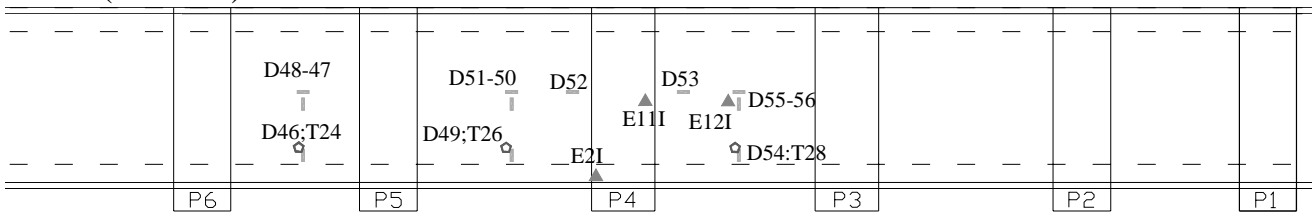

Legend:

$\Delta$ Strain sensors

- Temperature sensors

- Long gauge sensors

Displacement sensors in 'Z' configuration

- Displacement sensors

Figure 15. Monitoring plan of the Vila Fria bridge (fiber optic sensors).

Displacement sensors were installed between stone blocks where displacements are expected to be maximal. The LPDSs applied along the arches in a $\mathrm{Z}$ configuration are meant to register relative movements both in longitudinal and transverse directions. Realizing how stone blocks move and rotate makes it better to understand the global behavior of such structures. Displacement sensors were also placed underneath the arch to monitor block interfaces. They are both in longitudinal and transverse positions along the bridge axis and transversely on the joints between the first row of blocks and the others approximately bellow the border between timpani walls and filling material. 
The four Long Gauges placed on the bridge allow monitoring the relative displacements between timpani walls. Three of them are installed above the pillars where the wall is deeper and the other one is placed near the abutment to understand how it influences the timpani wall behavior.
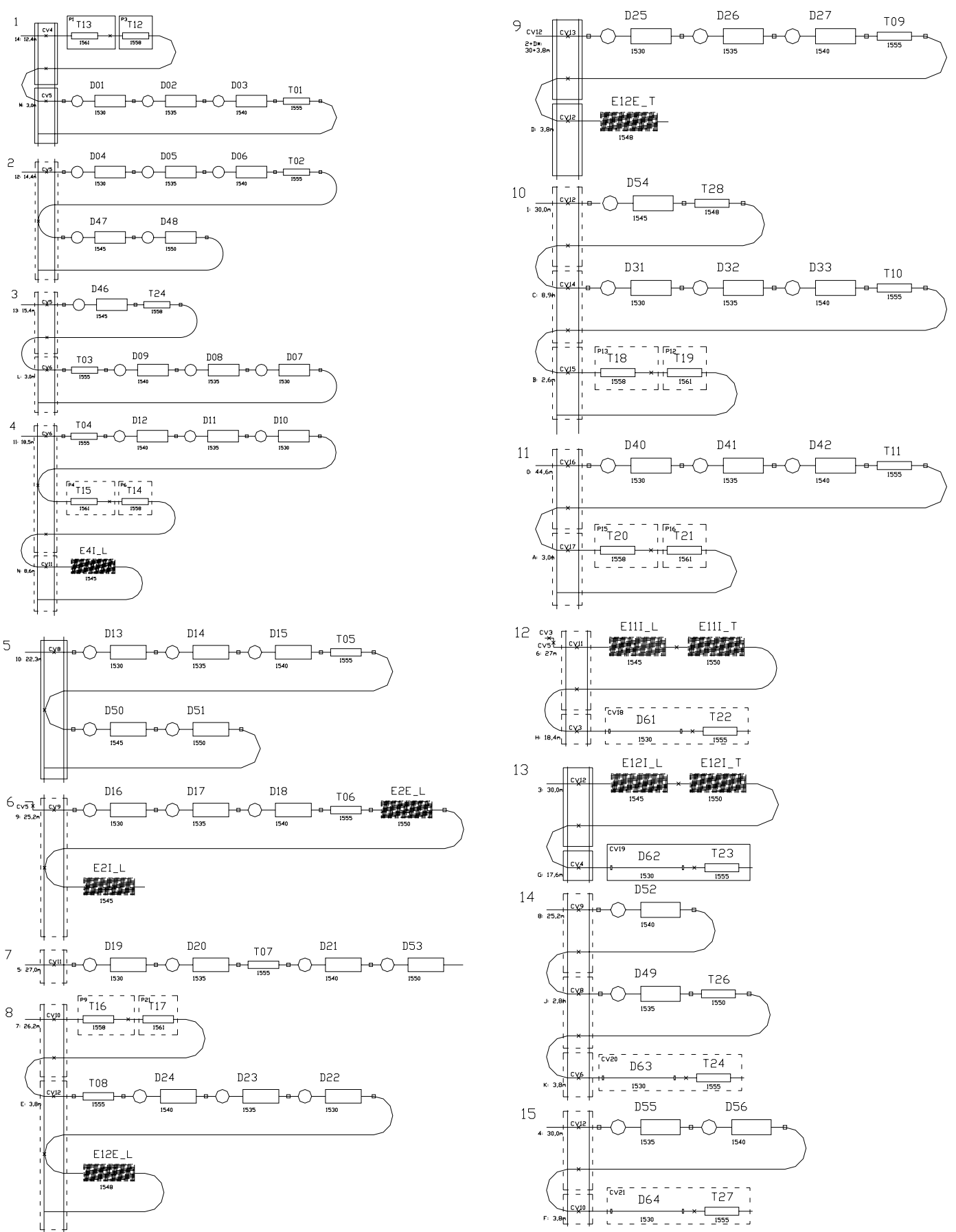

Legend:

LPDS TS CSG $\quad$ TS

Figure 16. Fiber optic sensing network branches. 
The fiber optic strain sensing network, together with the conventional strain gauges, is expected to give the stress distribution along the arch thickness. Strain is monitored both on the upper and underside of the selected blocks. Stress is achievable using Hooke's Law and the experimentally obtained Young Modulus for the used stone, because stress levels are sufficiently low to allow assuming linear elastic behavior.

The temperature sensing network was defined based on the monitoring project of the sensors to be corrected.

The optical network architecture was also defined taking into account the particularities and real placement of sensors in order to optimize the number and the length of fiber optic cables to be deployed. For example, Long Gauges were placed at the end of the branches because these sensors are terminal. Another important issue consists on the definition of the central wavelength for each sensor. This had to be carefully studied taking into account expected measurement ranges for each sensor in order to maximize multiplexing capacity while avoiding cross-talk. All fifteen branches are schematically represented in Figure 16.

\subsection{Optical cabling and cabinet}

In total, approximately $800 \mathrm{~m}$ of optical fibers were deployed along the bridge. The distribution of fiber optic cables along the bridge, from the measurement unit to the sensors, was made through a metallic duct connected to plastic pipes at selected locations. The same infrastructure was used for electrical cabling. Figure 17 (a) shows the duct and some of the connections during the installation of the complete monitoring system. Figure 17 (b) illustrates the amount of fiber optic cables that were used in this project. Two different methods were used to join fibers in the Vila Fria bridge: fusion splice and butt-joint connections. To reduce losses, all the joints inside the duct are fusion splices. Nevertheless, because this type of joints is much more complicated to perform in situ, butt-joints were used for the groups of three LPDSs in Z configuration together with the associated temperature sensor. Another reason is that these connections also simplify the replacement of sensors when necessary. Figure 18 (a) is a picture taken during the execution of a fusion splice. Figure 18 (b) shows one connection using adapters.
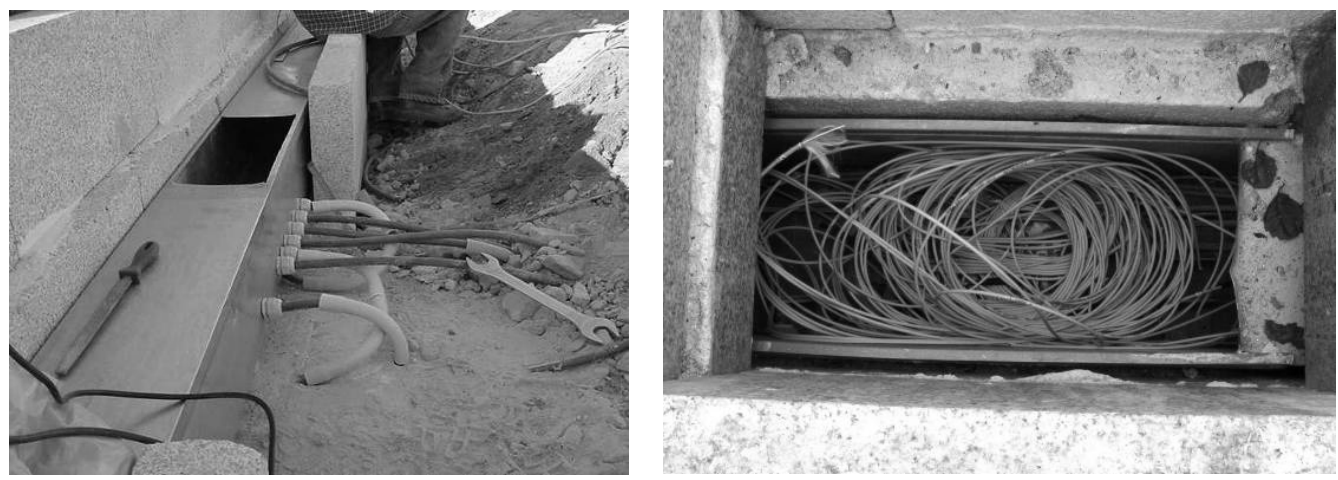

Figure 17. Distribution of fiber optic cables along the bridge: (a) metallic duct; (b) fiber optic cabling.
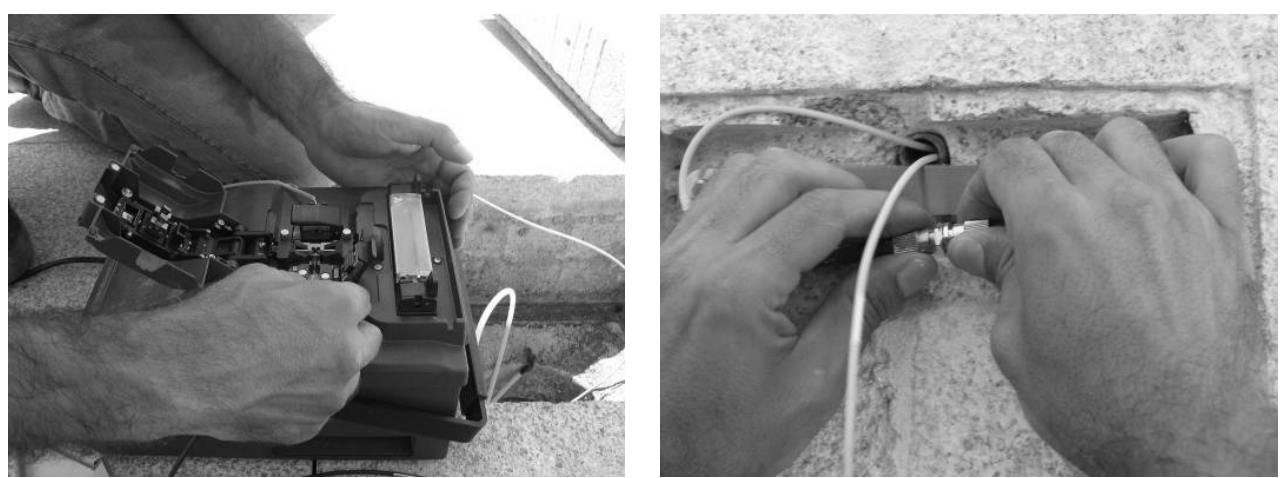

Figure 18. Coupling: (a) fusion splice; (b) butt-joint connection. 
For proper protection to the demanding environmental conditions near the river, the measurement unit was accommodated in a cabinet together with other equipments related with the conventional sensors used in the monitoring of the Vila Fria bridge. Figure 19 shows the cabinet being implemented.

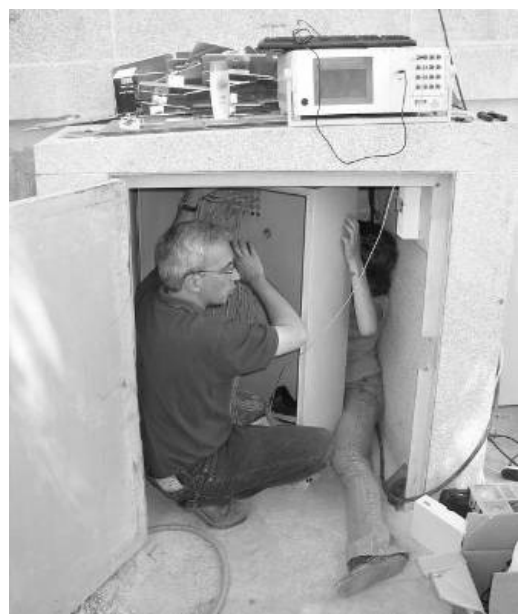

Figure 19. Implementation of the cabinet used to accommodate the equipment.

\subsection{Installation issues}

One of the major problems concerning the installation of such a monitoring network is the fact that it has to be prepared and executed in the construction site and while construction work is being carried out. In fact, most sensing components are extremely delicate and sensible to dirt and dust. For example the metallic parts used for fixing and protecting the invar wire had to be installed while timpani walls were still uncovered from the filling material. This meant that when installing the Long Gauge, screw heads and stainless steel protection tube were all dirty. Another issue found relating to Long Gauges installation was the fact that they were placed deep relatively to the available opening. Figure 20 (a) reveals the depth of the fixing parts of the Long Gauge and Figure 20 (b) shows the wrench used to ease the operation.
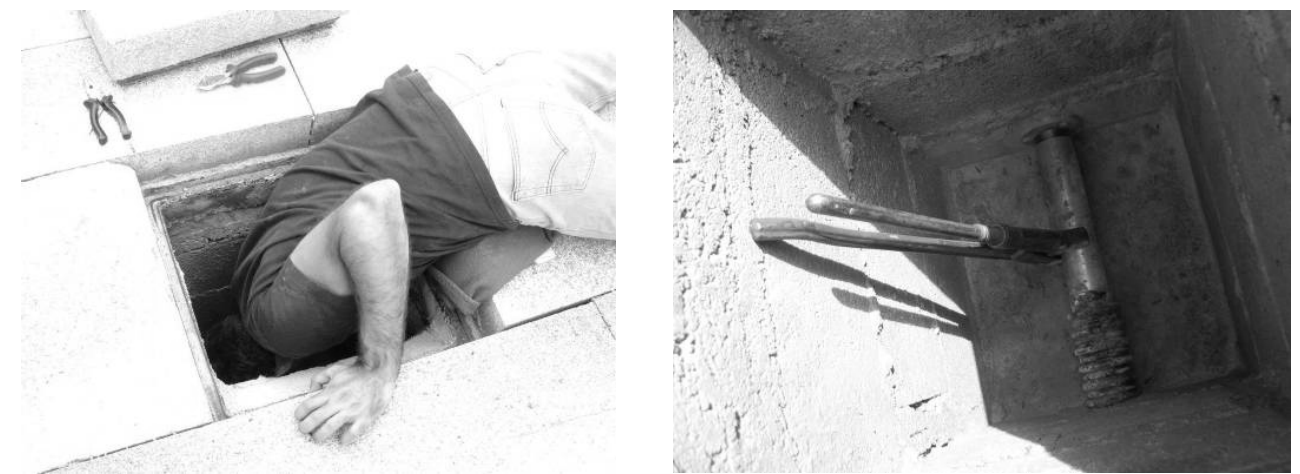

Figure 20. Installation of invar wire for Long Gauges: (a) access to the sensors; (b) used wrench.

Another difficulty was related with the installation of the LPDSs. For aesthetic reasons, the cavities prepared to accommodate these sensors were as small as possible, which constrained their placement and also the management of optical fibers, butt-join connections and associated temperature sensors (Figure 21 (a)). This, together with the fact that access was not easy and that some of the sensors had to be installed facing down on the arches intrados, as shown in Figure 21 (b), is a sample of the difficult and tricky issues that have occurred during installation process. 

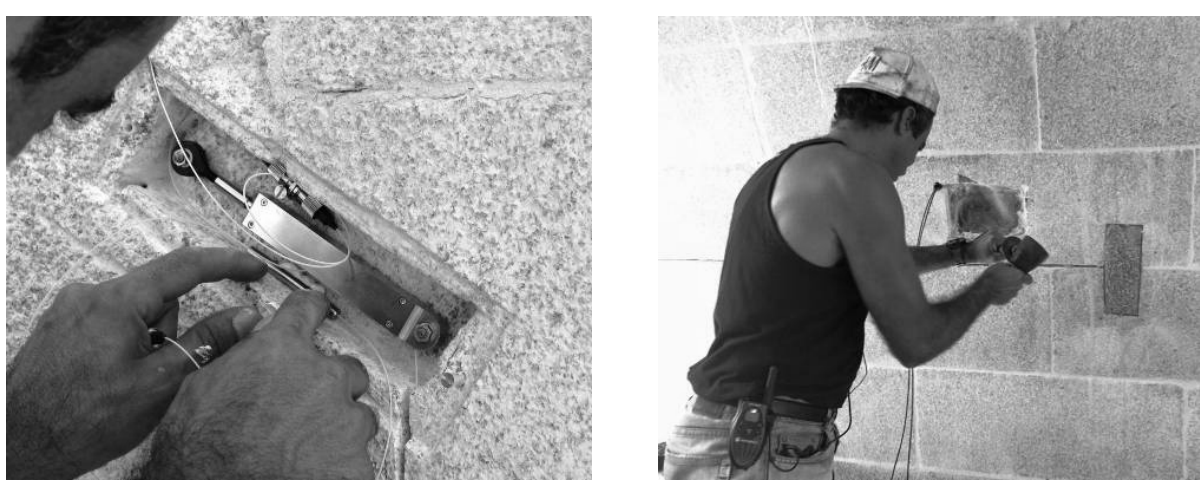

Figure 21. Installation issues: (a) LPDSs in small cavities; (b) composite strain sensors in the intrados.

\subsection{Network testing and operation}

A complete test was run out to the installed fiber optic sensing network, and it was possible to verify that it provides data relative to all the sensors at the predicted rate when all the fiber optic branches are connected to the measurement unit. Also, by checking each branch individually, it was possible to verify that all were equilibrated in terms of power budget and fully functional in terms of sensors. As an example, Figure 22 shows the spectrum reflected by the sensors in branch 8 as measured by the BraggMeter. This same branch was measured again three months later, and the result is shown in Figure 23. From this figure, it can be seen that no extra losses were induced by the environmental conditions (the residual reflection floor visible in Figure 23 is due to an unclean connector that was used during an in situ routine test).

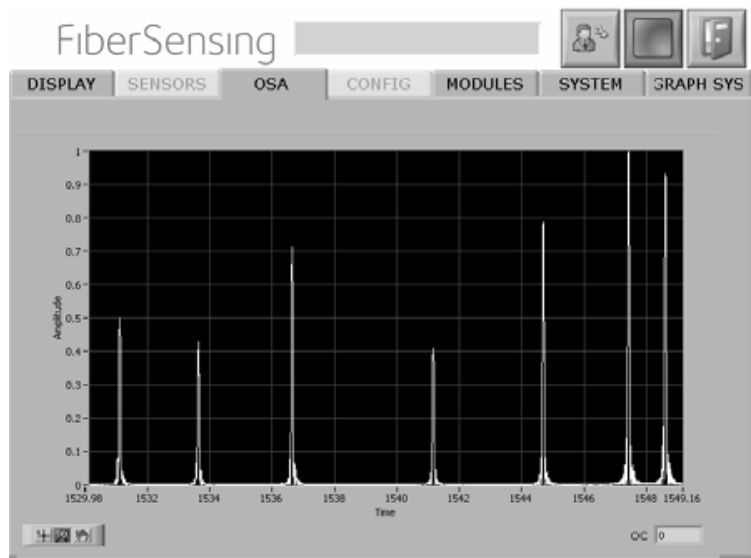

Figure 22. Example of spectrum reflected from sensing branch 8 measured with BraggMeter.

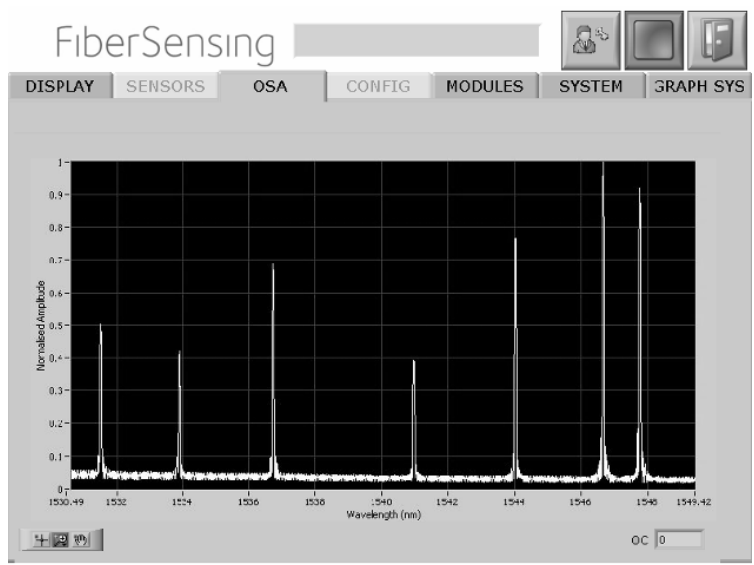

Figure 23. The same branch as in Figure 22 but obtained three months later. 
The remote transmission module was also tested. It is presently managing the transmission of the measured data both from the BraggMeter and the conventional data takers over a standard GPRS communication protocol to a remote computer placed in the Faculty of Engineering, University of Porto. This system will soon start continuous operation in order to update a website completely dedicated to the real time observation and analysis of monitoring data relative to the Vila Fria bridge. It is also expected that the results relative to the general load test will be available at the website shortly after its completion, which is expected to happen within the next few weeks.

\section{CONCLUSION}

In conclusion, the fiber optic sensing network installed on the Vila Fria stone masonry bridge, which is composed by 85 sensors and more than $800 \mathrm{~m}$ of fiber cables, is fully functional and ready to remotely deliver the relevant monitoring data. Sensors specially developed for this project demonstrated a good performance in laboratory tests, and it was verified to keep their characteristics five months after the installation. The interrogation of this sensing network with a single measurement unit was also demonstrated, as well as the archiving and transmission of data over a GPRS connection. It is expected to have all this data available very soon in a website that will allow real time analysis of this peculiar "live laboratory" bridge.

\section{ACKNOWLEDGEMENTS}

The authors would like to thank Teresa Restivo from the DEEC-FEUP, the LESE staff (Daniela Gloria and Valdemar Luís), Filipe Ferreira from the Contractor, and Marta Girão and Filipe Sá from FiberSensing for their valuable contribution to this work.

\section{REFERENCES}

1 Raman Kashyap, Fiber Bragg Gratings, Academic Press, San Diego, 1999.

2 Edited by E. Udd, Fibre Optic Smart Structures, John Wiley \& Sons, New York, 1995.

3 Edited by F. Ansari, Fibre Optic Sensors for Construction Materials and Bridges, Technomic Publishing, Lancaster, 1998.

4 Edited by J. M. López-Higuera, Handbook of Optical Fibre Sensing Technology, John Wiley \& Sons, Chichester, 2002.

5 Edited by F. Ansari, Sensing Issues in Civil Structural Health Monitoring, Springer, Netherlands, 2005.

6 A. Arêde, A. Costa, C. Costa, C. Barbosa, P. Costa, Monitorização do Comportamento Estrutural de uma Ponte Nova em Arcos de Alvenaria de Pedra, Proceedings of $6^{\circ}$ Congresso Nacional de Mecânica Experimental, Ponta Delgada, 2005.

7 S. Fischer, B. Verwilghen, M. Voet, M. Jobmann, F. Glötzl, M. Bugaud, P. Ferdinand, Fibre Bragg Grating Sensors and Sensing Systems for Civil Engineering and Mine Applications, OFS-13, 13th International Conference on Optical Fiber Sensors, April 12-16, 1999, Kyongju, Korea..

8 M. Jobmann, S. Fischer, B. Verwilghen, M. Voet, Fibre Optic Monitoring Systems for Operational Safety Requirements of Underground Waste Disposal Sites, Distec'98, International Conference on Radioactive Waste Disposal, 9-11 September 1998, Hamburg, Germany. 